\title{
A Visual Detection System for Determining Tritium Surface Deposition Employing Phosphor Coated Materials
}

\author{
C. A. Gentile, S. J. Zweben, C. H. Skinner, K. M. Young, J. Parker, L. Ciebiera, \\ S. W. Langish, A. von Halle, C. W. Kennedy \\ Princeton Plasma Physics Laboratory, Princeton University, Princeton, New Jersey, 08543 USA \\ K. Isobe \\ Japan Atomic Energy Research Institute, \\ Tritium Engineering Laboratory, Tokai, Ibaraki 319-1195, Japan
}

\begin{abstract}
A method for visually observing tritium deposition on the surface of TFTR D-T tiles is being investigated at PPPL. A green phosphor (P31, zinc sulfide: copper) similar to that used in oscilloscope screens with a wavelength peak of $530 \mathrm{~nm}$ was positioned on the surface of a TFTR D-T tile. The approximately 600 gram tile, which contains approximately $1.5 \mathrm{Ci}$ of tritium located on the top $\sim 1-50$ microns of the surface, was placed in a two liter lexan chamber at Standard Temperature and Pressure (STP). The phosphor plates and phosphor powder were placed on the surface of the tile which resulted in visible light being observed, the consequence of tritium betas interacting with the phosphor. This technique provides a method of visually observing varying concentrations of tritium on the surface of D-T carbon tiles, and may be employed (in a calibrated system) to obtain quantitative data.
\end{abstract}

\section{Introduction}

A detector for visually observing tritium deposited on the surface of TFTR D-T limiter tiles has been developed at PPPL. The detector employs phosphor coated onto a thin glass plate or a mylar film matrix. The phosphor surface is positioned on or slightly $(\sim 1 \mathrm{~mm})$ above a tritiated carbon based limiter tile. Visual tritium detection occurs when tritium beta particles emitted from the surface of the tile[1] interact with the phosphor coated material. The surface of the TFTR limiter tiles contain varying concentrations of tritium which have been co-deposited [2].

The distribution of tritium on the surface of the tile has been found to be nonuniform, and requires analysis to understand the dynamic distribution of this material. The interaction of tritium beta particles with P31 phosphor leads to the generation of a visible light with a wavelength peak of 530 $\mathrm{nm}$ [3]. The employment of a visual detection method for determining tritium concentrations on the surface of materials is of considerable interest. Visual detection systems for tritium employing phosphor as the active medium provide a nondestructive, fast, reproducible, and inexpensive method for visually observing tritium concentrations on surfaces. Experimental results employing this configuration show a relatively strong production of photons, which were easily observed by the unaided (dark adapted) eye. An added benefit to this detection configuration is that it is not susceptible to gamma radiation in mixed radiation fields. 


\section{System Configuration}

A $\sim 600$ gram TFTR limiter tile (Fig. 1.) which contains $\sim 1.5 \mathrm{Ci}$ of tritium in the top $\sim 1-50$ microns [4] of the surface was placed in an air filled two liter cylindrically shaped lexan chamber at STP. On the surface of the tile were placed P31 phosphor coated plates with a zinc sulfide:copper composition.

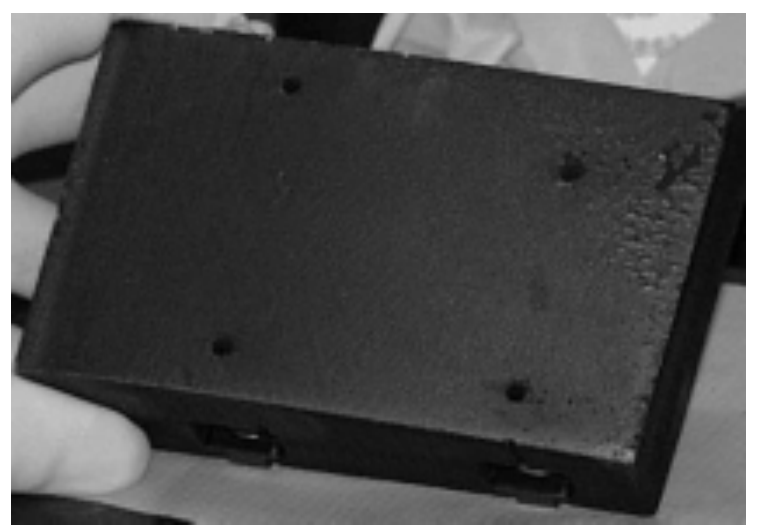

Figure 1. TFTR Limiter Tile

The chamber was sealed and placed in a dark room. After several minutes personnel in the room were able to observe visible light emanating from those sections on the tile where the phosphor had been placed (Fig. 2).

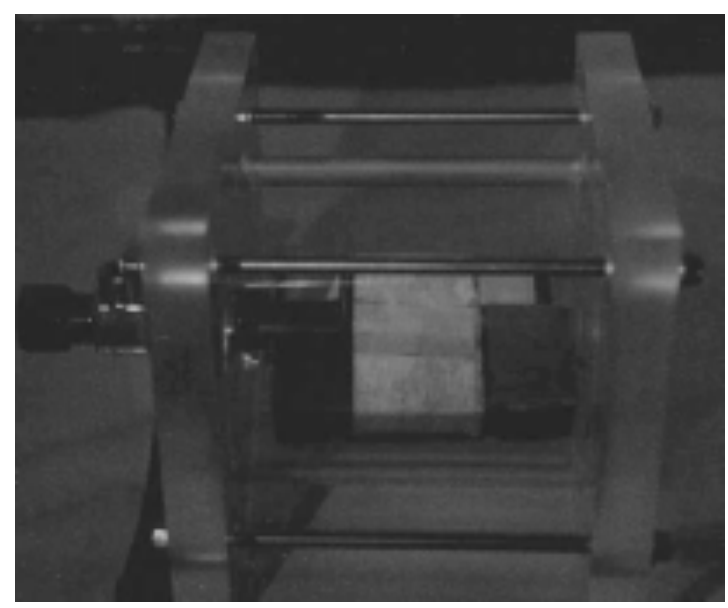

Figure 2. Lexan Observation Chamber
A control surface which employed a non-tritiated surface similar to that of the tile was also positioned in the room in a similar fashion with phosphor. The control was placed next to the lexan observation chamber and remained black with no signs of visible light during the extent of the experiment.

Currently a more sensitive (light imager) configuration is being fabricated, which is expected to visually detect much lower concentrations of tritium. The new system (Fig. 3) employs a highly sensitive charge-coupled device (CCD) [5] which is internally cooled to $10{ }^{\circ} \mathrm{C}$. The silicon based CCD (model KAF1400) imager is extremely sensitive to visible light. In crystalline silicon, the atoms are covalently bonded to form a lattice structure. Photons generated by the interaction of tritium beta particles on phosphor can penetrate the lattice and break these covalent bonds, thus generating an electric charge. Sensitivity of the system is further enhanced in that charge can be accumulated over an extended period of time (by increasing the exposure time). Using a system configuration which employs a CCD imager can greatly increase the sensitivity of the visual tritium detector. A CCD imager also has the advantage of being coupled to a computer, thus providing a system with potential for better quantitative measurements.

System calibration can be accomplished by configuring a tritium calibrated surface activity source under the phosphor plate. As is in the case with a tritiated tile, the beta particles impacting the phosphor fixed to the plate will generate visible light. The intensity of the light is proportional to the number of beta particles striking the phosphor which is a function of the tritium activity on the calibration source. Calibration surface activity sources are available from commercial vendors in 
various tritium concentrations and geometries.
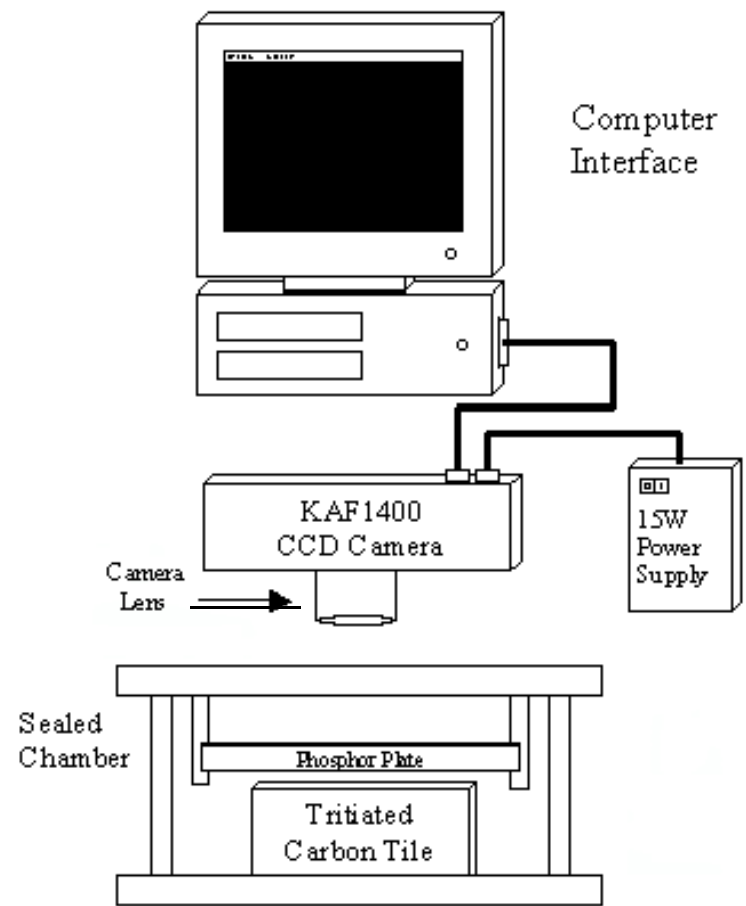

Figure 3. Visual Tritium Detection System

Operation of the CCD - computer based visual detection system employs a similar lexan observation chamber which was used in the first non CCD-computer configuration. The system is relatively easy to operate. Future plans include the fabrication of a light sealed box which will encase the sealed lexan chamber and lens of the KAF1400 CCD camera. In this configuration tile surface analysis for tritium deposition and concentration can be performed on the order of several minutes per tile.

Preliminary testing with the new system indicates that $\sim 24$ second exposure to the CCD camera will provide enough sensitivity to form clear images of the phosphor light emission excited by the tritium betas from the surface of TFTR tiles. In addition, since the CCD camera is interfaced to a computer, images can be compared, surfaces can be digitally mapped, and the extent of data analysis can be significantly increased.

\section{Conclusion}

The development of an optical surface tritium detection system employing phosphor provides a method to visually observe tritium deposition on the surfaces of TFTR limiter tiles, and possibly other materials. The employment of a CCD imager linked to a computer provides a tritium detection system that can be calibrated, and will significantly increase the ability to perform data analysis on the surface of materials which contain tritium.

\section{Acknowledgements}

The authors would like to acknowledge the efforts of the PPPL Health Physics staff for safely and efficiently controlling the radiological conditions associated with loading tritiated components into the visual detection chamber.

Funding for the development of the visual tritium detection system was provided by JAERI through the Annex IV to the JAERI/DOE Implementing Arrangement on Cooperation in Fusion Research and Development, and by USDOE Contract numbers DE-ACO2-76CH03073 and DEACO5-960R22464.

\section{References}

[1] S. J. Zweben, C. A. Gentile, D. Mueller, et al, "In-vessel tritium measurements using beta decay in the Tokamak Fusion Test Reactor", Review of Scientific Instruments 70, pgs. $1119-1122$ (1996).

[2] Gentile, C. A., Skinner, C. H., Young, K. M., Nishi, M., Langish, S., et al, Insitu Tritium Measurements of the 
Tokamak Fusion Test Reactor Bumper Limiter Tiles Post D-T operations. Presented at the $5^{\text {th }}$ International Symposium on Fusion Nuclear Technology Rome, September 19-24, 1999.

[3] Phosphor Resource Manual for Industrial and Military Cathode Ray tubes, Section 3 Standard Phosphors.

[4] Skinner, C. H., Gentile, C. A., Menon, N. M., Barry, R. E., "Flaking of codeposited hydrogenated carbon layers on the TFTR Limiter". Nuclear Fusion, Vol. 39, No. 9 (1999).

[5] I. Youle, A. A. Haasz, "Profiling with tritium imaging", Journal of Nuclear Materials 248, pgs. 64 - 71 (1997). 